\title{
ESTILO DE VIDA DOS ESTUDANTES DE ENFERMAGEM DE UMA UNIVERSIDADE DO INTERIOR DE MINAS GERAIS*
}

Rafael Damacena de Oliveira Pereira Soares ${ }^{1}$, Luciana de Freitas Campos ${ }^{2}$

RESUMO: Os objetivos deste estudo foram caracterizar o entendimento sobre o estilo de vida dos estudantes de enfermagem de uma Universidade do interior de Minas Gerais e analisar se há mudanças ocorridas em seus próprios estilos de vida durante o período letivo, através de uma pesquisa exploratório-descritiva. Os participantes foram os alunos de enfermagem da instituição. Foram analisados 87 questionários de auto-preenchimento contendo questões semi-estruturadas. A análise das respostas procedeu-se pelo número de ocorrências que o tópico apareceu. O entendimento dos graduandos por estilo de vida evidenciou o modo como se vive e sobre estilo de vida saudável, a qualidade de vida. Apontam como seu estilo de vida não-saudável o sedentarismo e saudável a alimentação, tópico também destacado quanto a mudanças no seu estilo de vida durante o período letivo. Sugere-se que os acadêmicos repensem seu estilo de vida e que desenvolvam ações que permitam a melhoria do mesmo.

PALAVRAS-CHAVE: Estilo de vida; Estudantes; Enfermagem.

\section{NURSING STUDENTS’ LIFESTYLE FROM A UNIVERSITY IN THE INTERIOR OF MINAS GERAIS-BRAZIL}

ABSTRACT: This study aimed to feature the understanding of nursing students' lifestyle from a University in the interior of Minas Gerais, Brazil, as well as to analyze if changes occurred in their own lifestyles during the school year by means of exploratory-descriptive research. The participants were nursing students from the institution. Eighty-seven self-applied questionnaires with semistructured questions were analyzed. The answers were analyzed through the number of times the topic appeared. The students' understanding of lifestyle evidenced how they live, while their understanding of healthy lifestyle evidenced quality of life. They pointed out sedentariness as their unhealthy lifestyle and their eating habits as their healthy one, which was also highlighted in terms of changes in their lifestyle during the school year. The students should reconsider their lifestyle and develop actions that allow improvements.

KEYWORDS: Lifestyle; Students; Nursing.

\section{ESTILO DE VIDA DE ESTUDIANTES DE ENFERMERÍA DE UNA UNIVERSIDAD DE INTERIOR DE MINAS GERAIS-BRASIL}

RESUMEN: Los objetivos de este estudio fueron caracterizar la comprensión acerca de estilo de vida de estudiantes de enfermería de una universidad del interior de Minas Gerais, Brasil, y también analizar si ocurrieron cambios en eses estilos de vida durante el período lectivo, mediante una investigación exploratorio-descriptiva. Los participantes fueron los alumnos de enfermería de la institución. Fueron analizados 87 cuestionarios de autorelleno con preguntas semiestructuradas. El análisis de las respuestas se efectuó mediante cuantas veces el tópico apareció. Lo que los graduandos comprenden por estilo de vida evidenció el modo como se vive y por estilo de vida saludable la calidad de vida. Indican como su estilo de vida no-saludable el sedentarismo, y saludable, la alimentación, tópico también destacado a respecto de cambios en su estilo de vida durante el período lectivo. Se sugiere que los académicos repiensen su estilo de vida y que desarrollen acciones que permitan la mejoría de este.

PALABRAS CLAVE: Estilo de vida; Estudiantes; Enfermería.

*Artigo extraído do Trabalho de Conclusão de Curso de Graduação em Enfermagem apresentado ao Departamento de Enfermagem da Universidade Federal dos Vales do Jequetinhonha e Mucuri-UFVJM. Diamantina-MG.

${ }^{1}$ Discente do Curso de Graduação em Enfermagem do Departamento de Enfermagem da UFVJM.

${ }^{2}$ Mestre em Enfermagem Fundamental. Docente do Departamento de Enfermagem da UFVJM. Orientadora do trabalho.

Autor correspondente:

Luciana de Freitas Campos

Praça Prof. José Augusto Neves, 43 - 39.1000 - Diamantina-MG Recebido: 04/12/07

E-mail: camposlf@gmail.com 


\section{INTRODUÇÃO}

Um estilo de vida conduzido por hábitos saudáveis é necessário para a manutenção da saúde física, mental e espiritual da pessoa, sendo um dos aspectos que colaboram para uma satisfatória qualidade de vida. A Organização Mundial da Saúde define qualidade de vida como

a percepção do indivíduo de sua posição na vida, no contexto da cultura e sistemas de valores nos quais vive e em relação aos seus objetivos, expectativas, padrões e preocupações $^{(1: 532)}$.

Entretanto, medir qualidade de vida é complexo pelo fato de não encontrar definição consensual sobre o que ela realmente significa, uma vez que depende da avaliação que as pessoas fazem ${ }^{(1-2)}$.

Ressalta-se que o processo saúde-doença é multifatorial e complexo, configurando processos compreendidos como um continuum, relacionados aos aspectos econômicos, socioculturais, à experiência pessoal e estilos de vida(3).

Durante as diversas fases do desenvolvimento humano, as pessoas apresentam alteração no entendimento da qualidade e mudanças em seu estilo de vida que podem ou não comprometer sua saúde.

Os meios de comunicação, bem como algumas ações dos serviços de saúde e de educação, têm demonstrado a preocupação das pessoas com sua saúde, evidenciando a importância e necessidade da adoção de estilo de vida saudável, como a preocupação com alguns hábitos como a dieta balanceada, o exercício físico, as práticas de meditação, sono/repouso e lazer.

Com relação aos estudantes do ensino fundamental e médio tem-se observado, empiricamente, a preocupação com fatores de risco relacionados à obesidade e hipertensão e à discussão de obtenção de estilo de vida saudável. Com relação aos estudantes universitários, observa-se que a atenção à saúde desta população está ainda não ocorre de modo efetivo, uma vez que este não se enquadra em nenhum grupo de atenção dos já estabelecidos pelos Serviços Básicos de Saúde, entretanto existem estudos que demonstram o interesse desta população pelo tema saúde( ${ }^{(4)}$.

Refletindo sobre aspectos relacionados ao estilo de vida, reportamo-nos ao cotidiano da população universitária e, da observação empírica da prática, evidenciam-se situações como: carga horária extensa em sala de aula e campo de estágio, sendo que as atividades complementares são desenvolvidas em outro período, geralmente no horário de refeições, à noite, finais de semana e feriados; uso abusivo de álcool e outras drogas; reduzido tempo de sono/repouso; hábito alimentar insatisfatório; não realização de atividade física regular; ansiedade/angústia constante pela cobrança do desempenho acadêmico; adaptação à outra cidade; afastamento temporário da família, namorado e amigos. Destacamos que

o estudante ao ingressar na universidade, passa por situações de crises acidentais, uma vez que sai do seu ambiente familiar e se depara com um mundo desconhecido podendo viver vários conflitos ${ }^{(4: 6)}$.

Essas situações podem influenciar negativamente no estilo de vida do acadêmico.

Encontramos na literatura, e o relato veio ao encontro de algumas das nossas reflexões, que o graduando de enfermagem, durante a formação, se prepara em várias ações que integram esse trabalho, com competência técnica, dialógica e política, mas enfrenta situações de sofrimento que podem contribuir para seu processo de humanização ou para a banalização das mesmas ${ }^{(2)}$.

Diante do exposto, surge uma inquietação com relação ao estilo de vida do graduando de enfermagem durante o período de formação acadêmica. Assim, o presente estudo tem por objetivos caracterizar o entendimento dos estudantes de enfermagem de uma Universidade do interior de Minas Gerais sobre estilo de vida e identificar mudanças ocorridas em seus próprios estilos de vida durante o período letivo.

\section{METODOLOGIA}

Realizamos uma pesquisa exploratório-descritiva, de natureza qualitativa, que consiste na coleta e análise sistemática de materiais narrativos mais subjetivos, por meio de procedimentos nos quais a tendência é um mínimo de controle imposto pelo investigador ${ }^{(5)}$.

O estudo foi desenvolvido em uma Universidade de um município do interior de Minas Gerais. N e s s a investigação garantiu-se o cumprimento dos preceitos da Resolução196/96, da Comissão Nacional de Ética em Pesquisa/ Conselho Nacional de Saúde do Ministério da Saúde. De posse da autorização da Universidade em estudo para realização desta pesquisa, a mesma foi encaminhada ao Comitê de Ética em Pesquisa da UFVJM, sendo aprovada (Registro de número 092/ 07). No Termo de Consentimento Livre Esclarecido foi garantido o direito de o sujeito recusar a participar 
ou se excluir do estudo quando julgar necessário e foi garantido o direito de anonimato e sigilo do sujeito.

O curso de enfermagem tem 10 anos, com 30 vagas e uma entrada anual. Os 103 acadêmicos de enfermagem matriculados no segundo semestre de 2007, do primeiro ao quarto ano, foram convidados a participar desta pesquisa sendo que 87 concordaram em participar, 7 se recusaram e 9 não foram encontrados durante o período de coleta de dados. Dos 87 participantes, 25 foram do primeiro ano de graduação, 23 do segundo, 21 do terceiro e 14 do quarto ano. O critério de inclusão consistiu no aceite em participar do estudo.

Cada questionário respondido foi legendado por uma letra e um número, nos quais a letra corresponde ao ano da graduação e o número corresponde à quantidade de alunos que responderam o questionário por ano de graduação. Os questionários respondidos pelos graduandos do primeiro ano foram chamados de questionários $\mathrm{A}$, de $\mathrm{B}$ os respondidos pelos do segundo ano, de $\mathrm{C}$ os respondidos pelos do terceiro ano e de $\mathrm{D}$ os respondidos pelos graduandos do quarto ano.

Os dados foram coletados durante o mês de outubro de 2007, por meio de um questionário contendo questões abertas: $\mathrm{O}$ que você entende por estilo de vida? O que é estilo de vida saudável? Qual (is) aspecto (s) do seu estilo de vida que você considera saudável (is)? Qual (is) aspecto (s) do seu estilo de vida que você considera não-saudável (is)? Ocorreu mudança em seu estilo de vida durante o período em que está cursando a graduação de enfermagem? Quais?

Após a apresentação do Termo de Consentimento Livre e Esclarecido, concordância e assinatura do mesmo, o questionário foi aplicado aos acadêmicos, no final de aula de uma das disciplinas de cada ano letivo, em sala de aula e em campo de estágio, sendo preenchido por eles na mesma ocasião.

De posse do questionário respondido, procedeuse a análise dos dados, seguindo a ordem do roteiro: entendimento por estilo de vida saudável; conceito de estilo de vida saudável; aspectos do seu estilo de vida saudável e não-saudável; mudança no estilo de vida durante o período letivo. Primeiramente, procedeu-se a organização dos dados e foi elaborado um esquema de codificação, relacionando os principais tópicos investigados. Neste esquema, os dados foram analisados por conteúdo e foram criados arquivos conceituais para cada tópico, facilitando a revisão das áreas por assunto. Então, foram elaboradas generalizações a partir da leitura exaustiva do material buscando temas recorrentes nos dados. Assim, procurou-se unir as peças temáticas em um todo integrado procedendo-se a contagem da freqüência do número de vezes em que um determinado tema surgiu $^{(5)}$.

\section{RESULTADOS E DISCUSSÃO}

Apresentaremos a seguir os resultados obtidos a partir da coleta de dados, das cinco questões semiestruturadas norteadoras desta investigação, acerca do estilo de vida dos acadêmicos de enfermagem de uma Universidade do interior de Minas Gerais, segundo o entendimento dos mesmos. Os tópicos codificados apresentados nas tabelas correspondem ao número de ocorrências em que apareceram nas respostas.

Com relação ao entendimento dos acadêmicos por estilo de vida, foram codificados os tópicos apresentados na Tabela 1 que segue.

Tabela 1 - Entendimento sobre estilo de vida entre os estudantes de enfermagem de uma Universidade do interior de Minas Gerais. Diamantina, 2007

\begin{tabular}{lccccccccc}
\hline & $1^{\circ}$ ano & $\%$ & $2^{\circ}$ ano & $\%$ & $3^{\circ}$ ano & $\%$ & $4^{\circ}$ ano & $\%$ & Total \\
\hline Modo como se vive & 10 & 41,67 & - & - & 12 & 54,54 & 9 & 56,25 & 31 \\
Cotidiano & 5 & 20,83 & 9 & 43,00 & 4 & 18,18 & 5 & 31,25 & 23 \\
Maneira como a pessoa se relaciona & 5 & 20,83 & 9 & 43,00 & 1 & 4,55 & 1 & 6,25 & 16 \\
com o ambiente e outras pessoas & & & & & & & & & \\
Conjunto de atividades realizadas & 2 & 8,33 & - & - & 3 & 13,63 & - & - & 5 \\
Fazer as coisas que gosta & 1 & 4,17 & 3 & 14,00 & 1 & 4,55 & - & - & 5 \\
Qualidade de vida & 1 & 4,17 & - & - & - & - & 1 & 6,25 & 2 \\
Hábitos, valores e crenças & - & - & - & - & 1 & 4,55 & - & - & 1 \\
Total & 24 & 100,00 & 21 & 100,00 & 22 & 100,00 & 16 & 100,00 & \\
\hline
\end{tabular}


Evidenciamos que a categoria apontada como definição de estilo de vida foi à relacionada ao modo como se vive seguida do cotidiano. O tópico "modo como se vive” foi abstraído de respostas como:

\section{[...] modo como cada pessoa conduz sua vida (D10).}

[...] maneira como cada pessoa escolhe viver (A20).

[...] maneira como se vive, que apesar de pessoal, é influenciado pelo meio no qual se vive (C1).

Destacamos que houve nas respostas dos participantes uma conotação individual/pessoal para o entendimento de estilo de vida, não baseado em literatura.

O cotidiano refere-se a atitudes e situações que fazem parte do dia-a-dia particular de cada pessoa em relação a horários, hábitos como alimentação, vestuário, afazeres acadêmicos e domésticos, relacionamentos, enfim os acontecimentos recorrentes no decorrer dos dias e que caracterizam as atividades da vida diária. Isso vem ao encontro da literatura que o define como aquilo que acontece diariamente, comum a todos os $\operatorname{dias}^{(6-7)}$.

Tabela 2 - Entendimento sobre estilo de vida saudável entre os estudantes de enfermagem de uma Universidade do interior de Minas Gerais. Diamantina, 2007

\begin{tabular}{lccccccccc}
\hline & $1^{\circ}$ ano & $\%$ & $2^{\circ}$ ano & $\%$ & $3^{\circ}$ ano & $\%$ & $4^{\circ}$ ano & $\%$ & Total \\
\hline Qualidade de vida & 18 & 51,42 & 11 & 28,20 & 13 & 46,42 & 5 & 27,78 & 47 \\
Alimentação saudável & 6 & 17,15 & 11 & 28,20 & 5 & 17,86 & 4 & 22,22 & 26 \\
Relacionamento interpessoal & 6 & 17,15 & 4 & 10,25 & 4 & 14,28 & 3 & 16,66 & 17 \\
Não fazer uso de álcool, cigarros & - & - & 8 & 20,52 & 4 & 14,28 & 3 & 16,66 & 15 \\
e/ou outras drogas & & & & & & & & & \\
Sono/Repouso & - & - & 4 & 10,25 & 1 & 3,58 & 1 & 5,56 & 6 \\
Religiosidade & - & - & 1 & 2,58 & - & - & 1 & 5,56 & 2 \\
$\begin{array}{l}\text { Estar bem mentalmente, fisicamente } \\
\text { espiritualmente }\end{array}$ & 4 & 11,42 & - & - & - & - & - & - & 4 \\
Vida social movimentada & 1 & 2,86 & - & - & - & - & - & - & 1 \\
Disciplina & - & - & - & - & 1 & 3,58 & - & - & 1 \\
Viver com o mínimo de industrialização & - & - & - & - & - & - & 1 & 5,56 & 1 \\
possível & & & & & & & & & \\
Total & 35 & 100,00 & 39 & 100,00 & 28 & 100,00 & 18 & 100,00 & \\
\hline
\end{tabular}

O tópico que sobressaiu quanto ao entendimento de estilo de vida saudável pelos alunos de todos os anos foi a qualidade de vida em que, no agrupamento das respostas destaca-se:

\section{[...] rotina que preserva e promove a saúde (B11).}

[...] que traga prazer e qualidade de vida (B53).

\section{[...] que garanta a longevidade (C10).}

\section{[...] quando não traz malefícios (D71).}

Os participantes deste estudo compreendem por qualidade de vida, o que nos traz prazer e nos proporciona saúde e bem-estar físico, mental e espiritual. Todavia não encontramos na literatura um consenso referente ao tema qualidade de vida ${ }^{(1-2,8)}$.

Apesar de diferenças conceituais entre estilo e qualidade de vida entende-se que um estilo de vida conduzido por hábitos saudáveis é necessário para manutenção da saúde física, mental e espiritual da pessoa sendo um dos aspectos que leva a uma satisfatória qualidade de vida.

Uma vez respondido o entendimento de estilo de vida saudável, os acadêmicos foram levados a refletir sobre seu estilo de vida saudável sendo codificados os tópicos conforme Tabela 3. 
Tabela 3 - Referências quanto aos aspectos de estilo de vida saudável entre os estudantes de uma Universidade do interior de Minas Gerais. Diamantina, 2007

\begin{tabular}{lccccccccc}
\hline & $1^{\circ}$ ano & $\%$ & $2^{\circ}$ ano & $\%$ & $3^{\circ}$ ano & $\%$ & $4^{\circ}$ ano & $\%$ & Total \\
\hline Boa alimentação & 7 & 18,91 & 16 & 37,20 & 8 & 18,60 & 14 & 36,84 & 45 \\
Bom relacionamento interpessoal & 13 & 35,13 & 10 & 23,26 & 10 & 23,26 & 7 & 18,42 & 40 \\
Práticas de exercícios físicos & 7 & 18,91 & 5 & 11,62 & 7 & 16,27 & 4 & 10,52 & 23 \\
Sono/Repouso & 2 & 5,41 & 4 & 9,30 & 9 & 20,93 & 3 & 7,89 & 18 \\
Ter Lazer & 2 & 5,41 & 2 & 4,66 & 4 & 9,30 & 4 & 10,52 & 12 \\
Religiosidade & 2 & 5,41 & 2 & 4,66 & 2 & 4,66 & 2 & 5,27 & 8 \\
Estudos & 2 & 5,41 & 3 & 6,97 & 2 & 4,66 & 2 & 5,27 & 9 \\
Outros & 2 & 5,41 & 1 & 2,33 & 1 & 2,32 & 2 & 5,27 & 6 \\
Total & 37 & 100,00 & 43 & 100,00 & 43 & 100,00 & 38 & 100,00 \\
\hline
\end{tabular}

Ter uma boa alimentação apareceu 45 vezes na resposta dos acadêmicos quanto ao seu aspecto do estilo de vida saudável. Por serem estudantes universitários, esse dado pode representar um conhecimento prévio adquirido. Há literatura que trata dos benefícios proporcionados a saúde pela alimentação adequada ${ }^{(9)}$.

Outro aspecto apontado foi o relacionamento interpessoal, que nos faz refletir sobre a importância que os relacionamentos com os familiares, amigos e namorados exercem na vida dos alunos. Infere-se que para que um estilo de vida seja saudável, possuir relacionamentos estruturados, pode proporcionar prazer e conferir segurança aos alunos, o que promove os bons momentos e torna mais fácil superar os desafios das atividades da vida diária.
O relacionamento interpessoal interfere na vida social. A convivência entre os seres humanos é definida por relacionamentos que fazem a diferença entre sofrimento e bem-estar e definem como se constrói a vida social em seu cotidiano. Problemas nas relações interpessoais resultam em problemas nas relações sociais ${ }^{(10)}$.

Ressalta-se que alguns alunos são de outras cidades, o que torna importante a discussão dos relacionamentos interpessoais por se encontrarem distantes dos seus lugares de origem, exigindo a necessidade de adequação dos relacionamentos antigos e dos recentes.

Quanto aos aspectos do seu estilo de vida nãosaudável, apresenta-se a codificação na Tabela 4:

Tabela 4 - Aspectos do seu estilo de vida não-saudável segundo os estudantes de uma Universidade do interior de Minas Gerais. Diamantina, 2007

\begin{tabular}{lccccccccc}
\hline & $1^{\circ}$ ano & $\%$ & $2^{\circ}$ ano & $\%$ & $3^{\circ}$ ano & $\%$ & $4^{\circ}$ ano & $\%$ & Total \\
\hline Sedentarismo & 4 & 10,52 & 12 & $3-$ & 10 & 14,93 & 11 & $2-$ & 37 \\
Alimentação deficiente & 5 & 13,15 & 10 & 25,00 & 10 & 14,92 & 10 & 18,19 & 35 \\
Sono/Repouso & 10 & 26,32 & 6 & 15,00 & 9 & 13,44 & 4 & 7,28 & 29 \\
Consumo alcoólico & 2 & 5,27 & 3 & 7,50 & 9 & 13,44 & 7 & 12,72 & 21 \\
Estresse & 4 & 10,52 & 4 & $1-$ & 6 & 8,93 & 3 & 5,45 & 17 \\
Consumo de cigarros & 2 & 5,26 & 2 & 5,00 & 5 & 7,46 & 4 & 7,28 & 13 \\
Falta de lazer & 3 & 7,89 & 3 & 7,50 & 3 & 4,47 & 3 & 5,45 & 12 \\
Uso de outras drogas & 3 & 7,89 & - & - & 4 & 5,98 & 3 & 5,45 & 10 \\
Fatores psicológicos & 2 & 5,27 & - & - & 4 & 5,98 & 4 & 7,28 & 10 \\
Déficit no relacionamento Interpessoal & 2 & 5,27 & - & - & 4 & 5,98 & 3 & 5,45 & 9 \\
Dificuldades Financeiras & 1 & 2,64 & - & - & 3 & 4,47 & 3 & 5,45 & 7 \\
Total & 38 & 100,00 & 40 & 100 & 67 & 100,00 & 55 & 100,00 & \\
\hline
\end{tabular}


O número de ocorrências de respostas quanto aos aspectos não-saudáveis do seu estilo de vida evidenciou para os acadêmicos do $1^{\circ}$ ano os tópicos sono/repouso, alimentação, sedentarismo e estresse; para os alunos do $2^{\circ}$ e $4^{\circ}$ ano o sedentarismo e alimentação; e para os estudantes do $3^{\circ}$ ano, sedentarismo, alimentação, sono/repouso e consumo alcoólico. As codificações apontadas como estilo de vida não-saudável que se destacaram em todos os anos do curso foram àquelas relacionadas ao sedentarismo seguido da alimentação, sono/repouso e consumo alcoólico.

Atentamos quanto à falta de atividade física, 0 sedentarismo entre os universitários. Embora haja conhecimento acadêmico sobre sua importância e necessidade, destaca-se o número de vezes (37) que aparece na fala dos alunos. Talvez isso possa ocorrer em detrimento da sobrecarga da carga horária em sala de aula e necessidade de despender seu tempo livre com atividades também relacionadas à universidade. A literatura nos traz que a participação em atividades físicas diminui expressivamente com o crescimento, especialmente da adolescência para o adulto jovem ${ }^{(11)}$.
Espera-se que haja mudança neste quadro, tendo em vista os benefícios que a prática de exercícios físicos promove em todas as fases do desenvolvimento humano.

A codificação relacionada à alimentação aparece tanto como um aspecto positivo quanto negativo do estilo de vida. Podemos inferir que os acadêmicos se contradizem e parece ter dificuldade em administrar 0 conhecimento adquirido na academia com o seu cotidiano, lembrando que o "cotidiano" foi um dos entendimentos do acadêmico quanto ao estilo de vida.

Por outro lado, entendemos que uma vez apresentando um estilo de vida não-saudável, um tópico passa a interferir no outro, como por exemplo, o sono/repouso interferir no estresse e em fatores psicológicos e, o uso abusivo de bebidas que contenham cafeína, durante a noite, interferir no padrão de sono/repouso. Assim, a avaliação individual do modo como o acadêmico conduz a sua vida sugere que necessita ser revista.

Com relação à mudança no estilo de vida durante o período letivo, foram codificados os tópicos apresentados a seguir:

Tabela 5 - Mudança no estilo de vida saudável segundo os estudantes de uma Universidade do interior de Minas Gerais. Diamantina, 2007

\begin{tabular}{lccccccccc}
\hline & $1^{\circ}$ ano & $\%$ & $2^{\circ}$ ano & $\%$ & $3^{\circ}$ ano & $\%$ & $4^{\circ}$ ano & $\%$ & Total \\
\hline Sim & 22 & & 22 & & 20 & & 15 & & 79 \\
Alimentação & 7 & 18,91 & 10 & 27,77 & 13 & 34,21 & 9 & 39,13 & 39 \\
Sono/Repouso & 9 & 24,32 & 9 & 25,00 & 7 & 18,42 & 6 & 26,08 & 31 \\
Lazer & 2 & 5,40 & 4 & 11,11 & 6 & 15,78 & - & - & 11 \\
Estresse & 5 & 13,51 & 1 & 2,77 & 3 & 7,89 & 1 & 4,34 & 10 \\
Estudos & 2 & 5,40 & 4 & 11,11 & 2 & 5,26 & 2 & 8,69 & 10 \\
Exercícios Físicos & 2 & 5,40 & 4 & 11,11 & - & - & 2 & 8,69 & 8 \\
Relacionamento Interpessoal & 4 & 10,81 & 1 & 2,77 & 2 & 5,26 & 1 & 4,34 & 8 \\
Universidade & 5 & 13,51 & - & - & - & - & - & - & 5 \\
Consumo alcoólico & - & - & - & & 3 & 7,89 & 2 & 8,69 & 5 \\
Mais responsabilidade & 1 & 2,70 & 1 & 2,77 & - & - & - & - & 2 \\
Consumo de cigarros & - & - & - & - & 1 & 2,63 & - & - & 1 \\
Menos informaçães a respeito do & - & - & 1 & 2,77 & - & - & - & - & 1 \\
mundo & & & & & & & & & \\
Menos tempo para questões religiosas & - & - & 1 & 2,77 & - & - & - & - & 1 \\
Dificuldade em estabelecer e cumprir & - & - & - & - & 1 & 2,63 & - & - & 1 \\
horários & & & & & & & & & \\
Total & 37 & 99,96 & 36 & 99,95 & 38 & 99,97 & 23 & 99,96 & \\
Não & 2 & & 1 & & 2 & & 3 & & 8 \\
\hline
\end{tabular}

Cogitare Enferm 2008 Jan/Mar; 13(2):227-34 
Ao responderem se houve mudança no seu estilo de vida, 79 acadêmicos referem que sim e o tópico alimentação foi uma codificação que apareceu do $1^{\circ}$ ao $4^{\circ}$ ano, por colocações como:

[...] minha alimentação piora, pois fico grande período sem comer durante o dia (A2).

[...] a alimentação fica desregulada, com refeições mais rápidas e menos saudáveis (C4).

[ ...] alimentação realizada em horários diferentes (B19).

[...] alimentação desequilibrada; passei a comer mais salgados, frituras, comidas com mais gordura (D14).

[...] não nos alimentamos direito às vezes, por falta de tempo (D13).

A literatura sugere que alterações nos hábitos alimentares podem estar relacionadas às aulas, aos trabalhos e aos estágios, que despendem tempo dos acadêmicos dentro e fora do campus da Universidade, dificultando se alimentarem em horários regulares e de forma satisfatória ${ }^{(12)}$.

Um aspecto relevante a ser considerado consiste na migração do aluno de sua cidade natal imprimindo uma outra realidade a ser vivenciada e administrada, exigindo adequações pessoais e profissionais às mudanças no seu estilo de vida, abstraindo falas como:

\section{[...] minha comida não é tão balanceada quanto em casa (C11).}

[...] e desde que me mudei para a cidade da Universidade meu estilo de vida mudou muito, inclusive na alimentação que é péssima (D12).

Os participantes relataram que após o ingresso na Universidade passaram a se alimentar de maneira menos saudável, talvez para economizar tempo no preparo da alimentação e diminuir o custo, refletindo na qualidade da alimentação.

O distanciamento da família, ao ingressar na universidade, tornar-se responsável pelas suas atitudes e por si próprio, encontrar pessoas de diferentes origens e consequentemente com hábitos diversos, podem ser explicações para essas alterações nos hábitos alimentares assim como, dificuldade financeira em proporcionar uma alimentação adequada ${ }^{(12)}$.

O tópico sono/repouso foi codificado a partir de repostas como: menos tempo de sono;o horário de dormir; sem tempo de dormir; horário de dormir e acordar. A literatura aponta que um padrão adequado de sono/repouso é fundamental para as pessoas ${ }^{(13)}$.

Um aspecto discutido em um estudo sobre sono/ repouso aponta que ele fica prejudicado, especialmente, no período letivo, com possível inversão de horários o que pode refletir em sua produção como graduando ${ }^{(13)}$.

A mudança no estilo de vida veio confirmar a observação empírica da prática, inquietação inicial deste estudo, sugerindo que os acadêmicos necessitam rever seus estilos de vida. Entretanto, não podemos afirmar que haja interação entre acadêmicos, docentes e a própria instituição universitária no que diz respeito a trabalhar os aspectos de promoção à saúde voltada a atitudes positivas que reforcem um estilo de vida saudável para os acadêmicos.

\section{CONSIDERAÇÕES FINAIS}

Este estudo permite afirmar que os estudantes de enfermagem têm um conceito próprio de estilo de vida (modo como se vive), estilo de vida saudável (qualidade de vida); pontuam seus estilos de vida saudáveis (alimentação) e não-saudáveis (sedentarismo) e identificam que há mudanças no seu estilo de vida durante o seu período letivo (alimentação) em especial frente a aspectos que podem ser prejudiciais à sua saúde.

Sugere-se como meio de minimizar as alterações negativas no estilo de vida do acadêmico, que a Universidade ofereça moradia e refeitório estudantis, intensifique oferta de bolsa de trabalho e iniciação científica e adequação na distribuição da carga horária das disciplinas por semestre letivo. Por sua vez, os estudantes devem se articular para pensar os aspectos do estilo de vida saudável no seu cotidiano.

A constatação de que os graduandos de enfermagem não adotam estilo de vida satisfatório, apesar de pontuarem maneiras que proporcionariam a adoção de um estilo de vida mais adequado evidencia a necessidade de mais estudos a esse respeito.

\section{REFERÊNCIAS}

1. Dantas RAS, Sawada NO, Malerbo MB. Pesquisas sobre 
a qualidade de vida: revisão de produção cinetífica das Universidades Públicas do Estado de São Paulo. Rev Lat-AmEnferm. 2003;11(4):532-8.

2. Saupe R, Nietche EA, Cestari, ME, Giorgi, MDM. Qualidade de vida dos acadêmicos de enfermagem. Rev Lat-Am Enferm. 2004;12(4):636-42.

3. Seidl EMF, Zannon CMLC. Qualidade de vida e saúde: aspectos conceituais e metodológicos. Cad Saúde Públ. 2004;20(2):580-8.

4. Figueiredo RM, Oliveira MAP. . Necessidades de estudantes universitários para implantação de um serviço de orientação e educação em saúde mental. Rev Lat-Am Enferm. 1995;3(1):5-18.

5. Polit DF, Hungler BP. Fundamentos de pesquisa em enfermagem. Porto Alegre: Artes Médicas; 1995.

6. Spink MJP. Pesquisando no cotidiano: recuperando memórias de pesquisa em Psicologia Social. Psicol e Socied. 2007;19(1):7-14.

7. Holanda AB. Mini Aurérilio Século XXI Escolar. O minidicionário da língua portuguesa. $4^{\mathrm{a}}$ ed. Rio de Janeiro: Nova Fronteira; 2001: p.790.

8. Kawakame PMG, Miyadahira AMK. Qualidade de vida de estudantes de graduação em enfermagem. Rev Esc Enferm USP. 2005;39(2):164-72.

9. Toral N, Slater B. Abordagem do modelo transteórico do comportamento alimentar. Ciênc Saúde Col. 2007;12(6):1641-50.

10. Leitão AP, Fortunato G, Freitas AS. Relacionamentos interpessoais e emoções nas organizações: uma visão biológica. RAP Rio de Janeiro. 2006;40 (5):883-907.

11. Alves JGB, Montenegro FMU, Alves RV, Oliveira FA. Prática de esportes durante adolescência e atividade física de lazer na vida adulta. Rev Bras Med Esporte 2005;(11)5:291-94.

12. Vieira VCR, Priore SE, Ribeiro SMR, Franceschini SCC, Almeida LA. Perfil socioeconômico, nutricional e de saúde de adolescentes recém-ingressos em uma universidade pública brasileira. Rev Nutr 2002;15(3):273-82.

13. Almondes KM, Araújo JF. Padrão do ciclo sono-vigília e sua relação com a ansiedade em estudantes universitários. Universidade Federal do Rio Grande do Norte. Est Psicol 2003;8(1):37-43. 